Math. Model. Nat. Phenom.

Vol. 7, No. 3, 2012, pp. 253-262

DOI: $10.1051 / \mathrm{mmnp} / 20127315$

\title{
Modeling Adaptive Behavior in Influenza Transmission
}

\author{
W. Wang* \\ Key Laboratory of Eco-environments in Three Gorges Reservoir Region, School of Mathematics and \\ Statistics, Southwest University, Chongqing, 400715, P. R. China
}

\begin{abstract}
Contact behavior plays an important role in influenza transmission. In the progression of influenza spread, human population reduces mobility to decrease infection risks. In this paper, a mathematical model is proposed to include adaptive mobility. It is shown that the mobility response does not affect the basic reproduction number that characterizes the invasion threshold, but reduces dramatically infection peaks, or removes the peaks. Numerical calculations indicate that the mobility response can provide a very good protection to susceptible individuals, and a combination of mobility response and treatment is an effective way to control influenza outbreak.
\end{abstract}

Keywords and phrases: mobility, response, pattern, stability

Mathematics Subject Classification: 34D20, 92D30

\section{Introduction}

Classical epidemic models do not consider behavior changes. For example, contact rates are assumed to be constant, which means that the population mobility is independent of infection risks. However, for some human diseases such as SARS and H1N1 influenza, people adapted their social behaviors to escape infection risks. Indeed, because they were lethal or threaten the health of human population significantly, schools were closed, conferences were canceled, and people reduced their visits to public places. These behavior changes can dramatically affect the progression of epidemic spread (see review paper [1] and the references cited therein).

A number of papers have formulated mathematical models to analyze influences of behavior adaptations on disease transmission. In [2], Capasso and Serio proposed a contact rate that is a decreasing function of disease prevalence. More general infection forces were investigated by Liu, Hethcote and Levin [11], Liu, Levin and Iwasa [12], Derrick and van den Driessche [4], and by Wang [22]. Ruan and Wang [15] studied the saturated contact rate of an epidemic model where saddle-node bifurcation, supercritical and subcritical Hopf bifurcations, and homoclinic bifurcation can occur and there exist two limit cycles. Sun, Yang, Arino and Khan [18] considered effects of media-induced social distancing on disease transmission in a two patch setting, Gao and Ruan [8] investigated the influences in the setting of $n$ patches.

\footnotetext{
${ }^{*}$ Corresponding author. E-mail: wendi@swu.edu.cn
} 
Behavior changes under influences of awareness of infection risk have been studied by papers $[5-7,9,13$, 19]. Mathematical models in these papers divide human population into a group with the lower infection risk and a group with the higher infection risk, describe the transfer of behavior trait as a consequence of social interaction, and analyze how these behavior responses affect disease transmission dynamics. They illustrate that behavior adaptations can induce rich dynamical patterns including sustained oscillations and multiple waves of infections.

Influenza is a contagious disease, which broke out during 1918-1920 (Spanish flu), 1957-1958 (Asian flu), 1968-1969 (Hong Kong flu), 2002-2003 (SARS) and 2009-2010 (A/H1N1), and killed tens of millions of people. Mathematical modeling of influenza has been proposed from different point of views by papers $[14,16,17,20,23]$. In the present paper, we consider influences of behavior changes on influenza spread. Note that there were quick infectivity information issued by authorities during the prevalence of those pandemic influenza. Under the influences of mass medium, people took protection measures such as using face masks or reducing social activity to decrease infection risk, and these strategies were very effective in controlling pandemic influenza. The objective of this paper is to formulate a mathematical model to simulate human mobility response to influenza infection, which is one of the most important behavior responses.

The organization of this paper is as follows. In the next section, I present the model formulation. Section 3 gives mathematical analysis of one system. In Section 4, numerical simulations are provided to reveal interesting patterns of disease transmission induced by mobility adaptations. The paper ends with a short discussion.

\section{Model formulation}

We consider two time scales. One is long enough so that we should include the natural birth and death of human population. The other one supposes that influenza spreads only seasonally. For the former, motivated by [14] we begin from the following framework:

$$
\begin{aligned}
& \frac{d S}{d t}=\mu N-\mu S-\lambda(t) S, \\
& \frac{d I}{d t}=\lambda(t) S-(\mu+\gamma) I, \\
& \frac{d R}{d t}=\gamma I-\mu R,
\end{aligned}
$$

where $S$ is the number of susceptible individuals, $I$ is the number of infectious individuals, $R$ is the number of recovered individuals, $N=S+I+R$ denotes the population size, $\mu$ is the per capita birth rate and death rate of the population and $\gamma$ is the sum of recovery rate and treatment rate. The infection force $\lambda(t)$ is given by

$$
\lambda(t)=p C \frac{I}{N},
$$

where $C$ is the contact number of one susceptible with all individuals per unit time, $C I / N$ gives the number of infectious contacts from those contacts, and $p$ is the valid transmission probability under $C I / N$ infectious contacts. Note that the increase of infection cases can drive people to use better protection measures such as wearing face masks, practicing better hygiene, and using preventive medicines during the outbreaks of influenza. This means that transmission probability $p$ is a decreasing function of infective number $I$. In the present paper, we assume that $p=p_{0} /(1+h I)$ with positive constants $p_{0}$ and $h$. Then

$$
\lambda(t)=C \frac{p_{0} I}{(1+h I) N} .
$$

Next, we assume that the contact number $C$ is a bilinear function of population size and intensity of population mobility, i.e., $C=k_{1} m N$ where $k_{1}$ is a proportional constant and $m$ denotes the intensity of 
population mobility, which could be the fraction of time to stay in public places in unit time. Then

$$
\lambda(t)=\frac{m k_{1} p_{0} I}{1+h I} .
$$

Note that $m$ is affected by infection risk and economic benefits of mobility. Without epidemic infections, mobility of population is only determined by economic benefits. We assume that the dynamics of $m$ are described by the Logistic equation:

$$
\frac{d m}{d t}=m(b-a m)
$$

which means that the social capacity of population mobility in the context of economics is $b / a$. With the occurrence of epidemics, the benefit of mobility is decreased by infection. We suppose that the loss rate of mobility benefit is proportional to the infection force: $w \lambda(t)$, where $w$ is a proportional constant and measures the human mobility response to infection risks. Then (2.2) is modified into

$$
\frac{d m}{d t}=m\left(b-a m-\frac{\alpha I}{1+h I}\right)
$$

where $\alpha=w k_{1} p_{0}$. Consequently, (2.1) incorporating the adaptive behavior of population mobility becomes

$$
\begin{aligned}
& \frac{d S}{d t}=\Lambda-\mu S-\frac{\beta m I}{1+h I} S, \\
& \frac{d I}{d t}=\frac{\beta m I}{1+h I} S-(\mu+\gamma) I, \\
& \frac{d R}{d t}=\gamma I-\mu R, \\
& \frac{d m}{d t}=m\left(b-a m-\frac{\alpha I}{1+h I}\right),
\end{aligned}
$$

where $\Lambda=\mu N$ and $\beta=k_{1} p_{0}$. Note that the population size $N$ is constant. It follows that $\Lambda$ is constant. Since the third equation of (2.4) is redundant, we remove it from (2.4) to obtain

$$
\begin{aligned}
& \frac{d S}{d t}=\Lambda-\mu S-\frac{\beta m I}{1+h I} S, \\
& \frac{d I}{d t}=\frac{\beta m I}{1+h I} S-(\mu+\gamma) I, \\
& \frac{d m}{d t}=m\left(b-a m-\frac{\alpha I}{1+h I}\right) .
\end{aligned}
$$

We now modify (2.5) to simulate the seasonal transmission of influenza. Because of sort time scale, we ignore the natural birth and natural death of human population and concentrate on disease transmission. Then we are lead to consider

$$
\begin{aligned}
& \frac{d S}{d t}=-\frac{\beta m I}{1+h I} S, \\
& \frac{d I}{d t}=\frac{\beta m I}{1+h I} S-\gamma I, \\
& \frac{d m}{d t}=m\left(b-a m-\frac{\alpha I}{1+h I}\right) .
\end{aligned}
$$

\section{Mathematical analysis}

We consider the mathematical analysis of $(2.5)$ in this section. $E_{0}=(\Lambda / \mu, 0,0)$ is a semi-trivial equilibrium that corresponds to the case where there is no population mobility. $E_{1}=(\Lambda / \mu, 0, b / a)$ is the disease-free equilibrium. 
By the computation method in [21], the basic reproduction number of $(2.5)$ is

$$
R_{0}=\frac{\Lambda \beta b}{\mu a(\mu+\gamma)}
$$

Set

$$
L_{*}=\frac{\mu a(\mu+\gamma)}{\beta b} .
$$

It is easy to see that $R_{0}<1$ is equivalent to $\Lambda<L_{*}$ and $R_{0}>1$ is equivalent to $\Lambda>L_{*}$. The next theorem gives the condition that influenza dies out.

Theorem 3.1. $E_{1}$ is globally stable if $R_{0}<1$.

Proof. By the first equation and the second equation of (2.5), we see that a nonnegative solution of (2.5) satisfies $S(t)>0, I(t) \geq 0$ and $m(t) \geq 0$ for $t>0$ and

$$
\limsup _{t \rightarrow \infty} S(t) \leq \frac{\Lambda}{\mu}, \quad \limsup _{t \rightarrow \infty} m(t) \leq \frac{b}{a} .
$$

Since $R_{0}<1$, it follows from the second equation of (2.5) that there exists an $\epsilon>0$ such that

$$
\frac{d I}{d t} \leq-\epsilon I
$$

Consequently, the Lyapunov-LaSalle theorem [10] implies that $E_{1}$ is globally stable.

We now consider the existence of positive equilibria in (2.5).

Theorem 3.2. There exists a unique positive equilibrium in (2.5) if $R_{0}>1$.

Proof. To find a positive equilibrium, we consider

$$
\begin{aligned}
& \Lambda-\mu S-\frac{\beta m I S}{1+h I}=0, \\
& \frac{\beta m S}{1+h I}-\mu-\gamma=0, \\
& b-a m-\frac{\alpha I}{1+h I}=0 .
\end{aligned}
$$

By the third equation, we obtain

$$
m=\frac{b+(b h-\alpha) I}{(1+h I) a} .
$$

Substituting it into the second equation of (3.2) and then solving $S$, we get

$$
S=\frac{a(1+h I)^{2}(\mu+\gamma)}{\beta(b+(b h-\alpha) I)} .
$$

Substituting (3.3) and (3.4) into the first equation of (3.2), we have

$$
b_{0} I^{2}+b_{1} I+b_{2}=0,
$$

where

$$
\begin{aligned}
& b_{0}=\left(\mu a h^{2}+\beta b h-\alpha \beta\right)(\mu+\gamma), \\
& b_{1}=(2 \mu a h+\beta b)(\mu+\gamma)-\beta(b h-\alpha) \Lambda, \\
& b_{2}=\mu a \gamma-\Lambda \beta b+\mu^{2} a .
\end{aligned}
$$

We consider two cases: 
(A) $b h \geq \alpha$.

(B) $b h<\alpha$.

Case (A) means that the response of population mobility to infection risk is limited when economic activity and daily life are more important. Case (B) implies that the mobility response to escape infection can be stronger.

When case (A) holds, we have $b_{0}>0$. It follows easily that (3.5) has a unique positive root if

$$
\Lambda>\frac{\mu a(\mu+\gamma)}{\beta b}=L_{*},
$$

which is equivalent to $R_{0}>1$.

When case (B) holds, it follows from (3.3) that a positive equilibrium $(S, I, m)$ of $(2.5)$ satisfies

$$
I<\frac{b}{\alpha-b h} .
$$

When

$$
b h<\alpha<\frac{h(\mu a h+\beta b)}{\beta},
$$

then $b_{0}>0$ and $b_{1}>0$. Set

$$
f(I)=b_{0} I^{2}+b_{1} I+b_{2}
$$

Then direct calculations indicate that

$$
f\left(\frac{b}{\alpha-b h}\right)=\frac{\mu a \alpha^{2}(\mu+\gamma)}{(b h-\alpha)^{2}}>0 .
$$

Since $R_{0}>1$ means $b_{2}<0$, it follows that there exists a unique root in $(0, b /(\alpha-b h))$ for $(3.5)$.

For the case where

$$
\alpha \geq \frac{h(\mu a h+\beta b)}{\beta},
$$

we have $b_{0} \leq 0$ and $b_{1}>0$. It follows from (3.10) and $R_{0}>1$ that there exists a unique root in $(0, b /(\alpha-b h))$ for (3.5). Note that (3.8) implies that one is forced to find root only in $(0, b /(\alpha-b h))$ to ensure the positivity in $m$ component of an endemic equilibrium. Consequently, there exists a unique positive equilibrium when $R_{0}>1$.

Note that the threshold $L_{*}$ for the existence of an endemic equilibrium is independent of $\alpha$, which reflects the response of adaptive mobility of human individuals to epidemic risks. Hence, the adaptation of mobility behaviors does not affect the epidemic threshold. However, we state that the adaptation can reduce the prevalence of infection.

Theorem 3.3. Let $\left(S^{*}(\alpha), I^{*}(\alpha), m^{*}(\alpha)\right)$ be a positive equilibrium of $(2.5)$. Then $I^{*}(\alpha)$ is a decreasing function of $\alpha$ when

$$
\alpha>\frac{(\mu+\Lambda h+\gamma)\left(\Lambda \beta b-2 \mu a \gamma-2 \mu^{2} a\right)}{\beta \lambda^{2}} .
$$

Proof. From the proof of Theorem 3.2, we see that $I^{*}$ is the unique positive solution of (3.5) when $\alpha \leq b h$ or (3.9) holds, and is the smaller positive root of (3.5) when (3.11) is valid. Thus,

$$
I^{*}(\alpha)=\frac{1}{2 b_{0}}\left(-b_{1}+\sqrt{b_{1}^{2}-4 b_{0} b_{2}}\right) .
$$

Simple algebraic computations lead to

$$
I^{*}(\alpha)=\frac{-2 b_{2}}{b_{1}+\sqrt{b_{1}^{2}-4 b_{0} b_{2}}} .
$$


If $\Delta(\alpha)=b_{1}^{2}-4 b_{0} b_{2}$, it follows from (3.6) that

$$
\Delta^{\prime}(\alpha)=\frac{1}{\beta}\left[2 \beta \Lambda^{2}+2(\mu+\Lambda h+\gamma)\left(2 \mu a \gamma-\Lambda \beta b+2 \mu^{2} a\right)\right],
$$

which is positive by (3.12). Furthermore, (3.6) indicates that $b_{1}$ is an increasing function of $\alpha$ and $b_{2}$ is independent of $\alpha$. Consequently, $I^{*}(\alpha)$ is a decreasing function of $\alpha$.

Let $E^{*}=\left(S^{*}, I^{*}, m^{*}\right)$ denote the unique positive equilibrium of $(2.5)$ when $R_{0}>1$. The following theorem shows that it is always stable when it exists.

Theorem 3.4. Let $R_{0}>1$. Then $E^{*}$ is asymptotically stable.

Proof. The Jacobian matrix at $E^{*}$ is

$$
J=\left[\begin{array}{ccc}
-\mu-\frac{\beta I^{*} m^{*}}{1+h I^{*}} & -\frac{\beta S^{*} m^{*}}{1+h I^{*}}+\frac{\beta S^{*} I^{*} m^{*} h}{\left(1+h I^{*}\right)^{2}} & -\frac{\beta S^{*} I^{*}}{1+h I^{*}} \\
\frac{\beta I^{*} m^{*}}{1+h I^{*}} & -\frac{\beta S^{*} I^{*} m^{*} h}{\left(1+h I^{*}\right)^{2}} & \frac{\beta S^{*} I^{*}}{1+h I^{*}} \\
0 & m^{*}\left(-\frac{\alpha}{1+h I^{*}}+\frac{\alpha I^{*} h}{\left(1+h I^{*}\right)^{2}}\right) & -a m^{*}
\end{array}\right] .
$$

Its characteristic equation in $\xi$ is

$$
\xi^{3}+p_{1} \xi^{2}+p_{2} \xi+p_{3}=0
$$

where

$$
\begin{aligned}
p_{1}= & \frac{I^{* 2} h\left(h a m^{*}+\mu h+\beta m^{*}\right)+I^{*} h\left(\beta S^{*} m^{*}+2 \mu+2 a m^{*}\right)+a m^{*}+\mu+\beta I^{*} m^{*}}{\left(1+h I^{*}\right)^{2}}, \\
p_{2}= & \frac{m^{*}}{(1+h I)^{3}}\left(a\left(1+h I^{*}\right)^{2}\left(\mu h I^{*}+\mu+\beta I^{*} m^{*}\right)\right. \\
& \left.+S^{*} \beta I^{*}\left(\beta I^{*} m^{*} h+I^{*} m^{*} h^{2} a+I^{*} \mu h^{2}+\beta m^{*}+a m^{*} h+\mu h+\alpha\right)\right), \\
p_{3}= & \frac{\beta S^{*} I^{*} m^{*}\left(\mu \alpha+\beta I^{*} m^{* 2} h a+\beta m^{* 2} a+\mu I^{*} m^{*} h^{2} a+\mu m^{*} h a\right)}{\left(1+h I^{*}\right)^{3}} .
\end{aligned}
$$

Clearly, $p_{1}>0$ and $p_{3}>0$. Direct calculations indicate that $p_{1} p_{2}-p_{3}$ is equal to

$$
\begin{aligned}
& \frac{m^{*}}{\left(1+h I^{*}\right)^{3}}\left\{\left(S^{*}\right)^{2} \beta^{2} I^{* 2} m^{*} h\left[h I^{*}\left(h a m^{*}+h \mu+\beta m^{*}\right)+h a m^{*}+h \mu+\beta m^{*}+\alpha\right]\right. \\
& +a\left(1+h I^{*}\right)^{3}\left(\mu h I^{*}+\mu+\beta I^{*} m^{*}\right)\left(\mu h I^{*}+a m^{*} h I^{*}+\mu+\beta I^{*} m^{*}+a m^{*}\right) \\
& +S^{*} I^{*}\left(1+h I^{*}\right) \beta\left[\left(I^{*}\right)^{2} h\left(h a m^{*}+h \mu+\beta m^{*}\right)^{2}+h\left(a m^{*}+\mu\right)^{2}+m^{*}(\beta \mu+a \alpha)\right. \\
& \left.\left.+I^{*}\left(2 h^{2}\left(a m^{*}+\mu\right)^{2}+h m^{*}\left(2 \beta a m^{*}+3 \beta \mu+a \alpha\right)+m^{*} \beta\left(\beta m^{*}+\alpha\right)\right)\right]\right\},
\end{aligned}
$$

which is positive. It follows from the Routh-Hurwitz conditions that $E^{*}$ is asymptotically stable.

\section{Simulations}

In this section, we use numerical calculations to find more interesting phenomena from population mobility adaptations. First, we consider (2.5). Motivated by [14], we take $\mu=0.00005, \gamma=0.2, \Lambda / \mu=10000$, which means that we consider a population of capacity 10000 and its average life span is 60, and the average infection period is 5 days. Then we fix $\beta=0.00025, b=2, a=1$ so that the valid contact number is 2 and the basic reproduction number is 12.5 when $m$ lies in its steady state. Let us take $h=0.01$. Then $I^{*}$ is a decreasing function of $\alpha$, which is shown in Figure 1. Note that the mobility adaptation cannot 


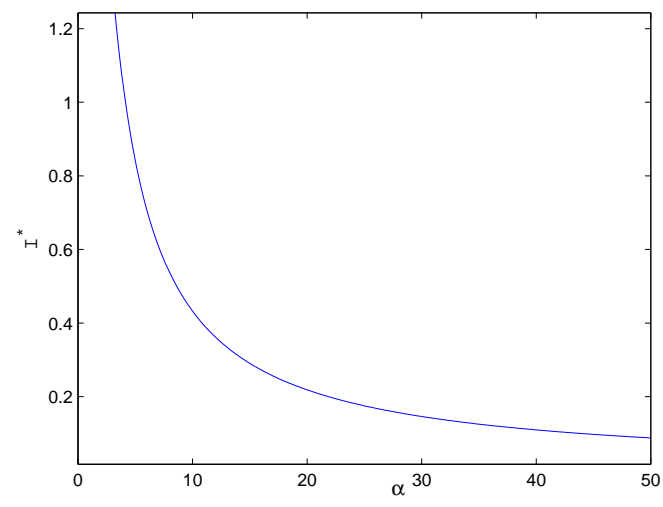

FIGURE 1. Mobility response reduces the infection level.
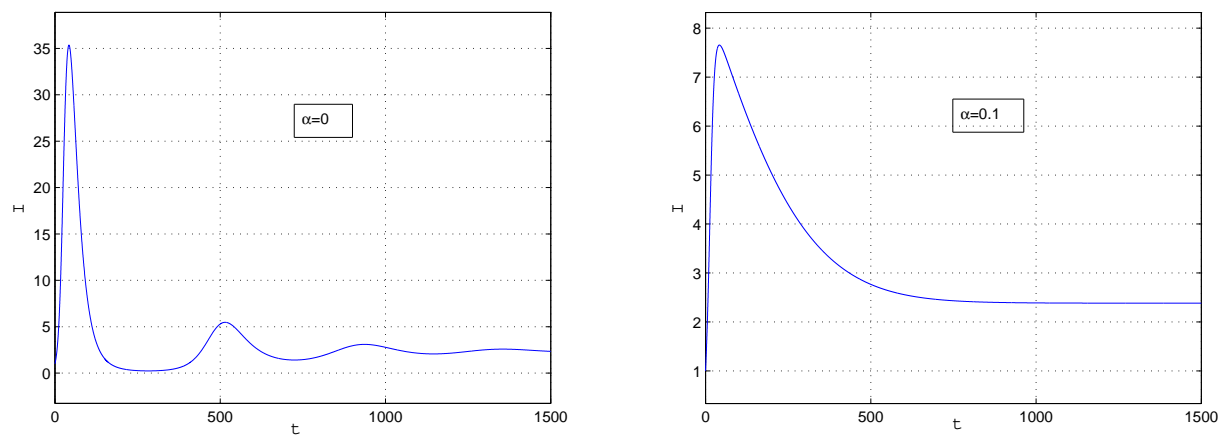

FiguRE 2. Mobility response reduces the infection level: the left panel gives dynamical changes of infected individuals without a mobility response where peak size is 35 , and the right panel shows the reduction of peak size which is less than 8 when the mobility response coefficient $\alpha$ equals 0.1 . The initial value of the solution is fixed by $(700,1,2)$. The time unit is a day.

eradicate the prevalence of influenza, but is helpful to control the disease. In fact, a strong adaptation of population mobility can drive epidemic prevalence to a low level. Then a suitable intensity of treatment, which increases the value of the $\gamma$, can eliminate the disease.

Now, we find effects of mobility adaptation on the pattern of disease spread. If $\alpha=0$, which means that there is no reduction in human mobility, then the left panel of Figure 2 indicates that $I(t)$ approaches a steady state in an oscillating way, where the first peak is much higher. Next, we increase $\alpha$ to 0.1 . Then the right panel of Figure 2 shows that there is only one peak and its magnitude is much reduced. When $\alpha$ is increased to 0.37 , the peak of infected individuals disappears and $I(t)$ grows monotonically to a steady state, as shown in Figure 3.

We now consider (2.6). Fix $\beta=0.00023, h=0.01, \gamma=0.2, b=2, a=1$ and consider a population of 1000. Without mobility response, the basic reproduction number is 2.3. For different response intensities $\alpha=0,0.05,0.1,0.2$, the left panel of Figure 4 shows that the mobility adaptations reduce the endemic peaks, whereas the stronger response leads to the larger infected numbers at the end of 120 days. The biological reason is that through the mobility response to infection risk, the number of infected individuals is decreased at a higher endemic level and more susceptible individuals are protected (see the right panel 


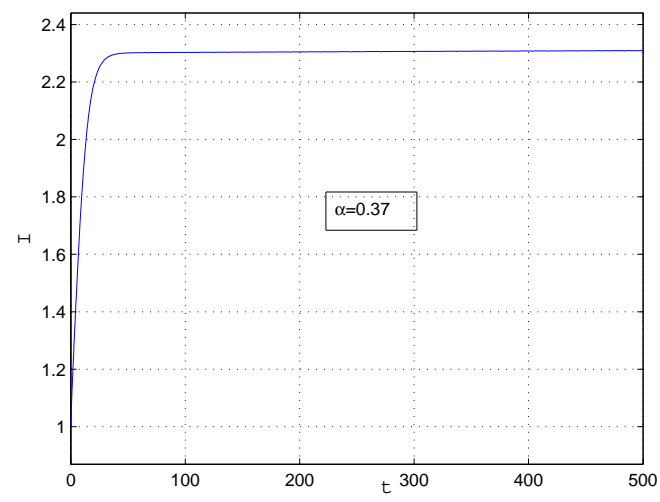

FIGURE 3. The infection peak disappears when $\alpha$ is increased to 0.37 , where the initial value of the solution is fixed by $(700,1,2)$. The time unit is a day.
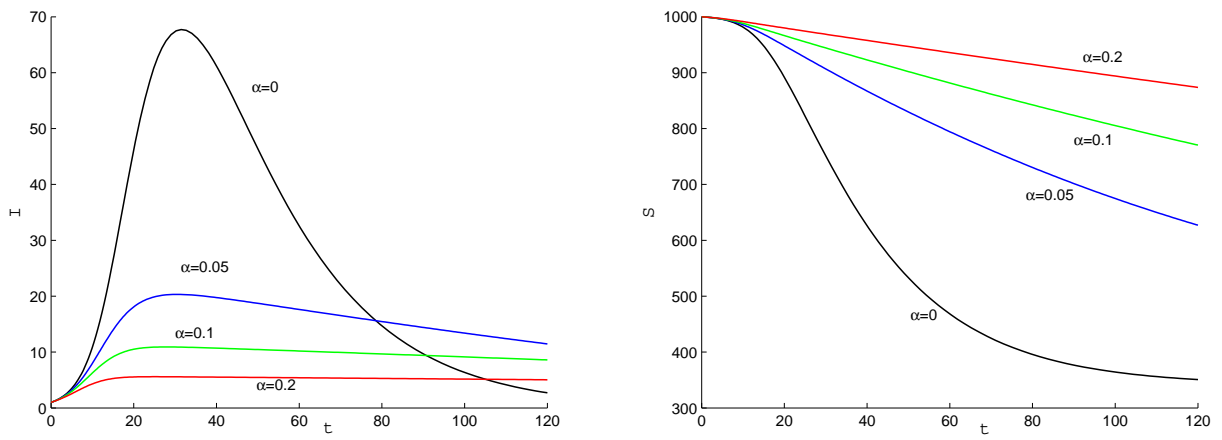

FigURE 4. Mobility response reduces endemic peaks and protects susceptible individuals: the left panel demonstrates the reduction of endemic peaks and the higher infected numbers at days 120 , the right panel shows that the adaptation protects susceptible individuals, where the initial value of the solution is fixed by $(1000,1,2)$.

of Figure 4). Then because mobility response does not influence the basic reproduction number, a larger susceptible pool leads to a higher infected numbers at days 120. Furthermore, the numbers of susceptible individuals at days 120 are 874,770,627,350, which correspond, respectively, to $\alpha=0.2,0.1,0.05$ and $\alpha=0$. Therefore, mobility responses to infection risk reduce significantly the transmission of influenza.

\section{Discussion}

In this paper, by introducing the variable of mobility intensity, which is regulated by economic benefits and infection risks, we have formulated two mathematical models that simulate the behavior response to infection risks. We have shown that the mobility adaptation does not affect the basic reproduction number which gives the invasion threshold of influenza. However, the mobility response can significantly reduce the epidemic prevalence so that an aid from treatment can eliminate the disease. Moreover, we have shown that the mobility response does not destroy the stability of system (2.5). Indeed, for constant $m$, it is well known that the disease-free equilibrium is globally stable if $R_{0}<1$ and the endemic equilibrium is globally stable if $R_{0}>1$. With the incorporation of adaptive mobility, Theorem 3.1 
means that the disease-free equilibrium is globally stable if $R_{0}<1$, Theorems 3.2 and 3.4 show that the endemic equilibrium is unique and is always stable when $R_{0}>1$. Extensive simulations also suggest that the endemic equilibrium is globally stable. This is in contrast with other studies $[3,5,15,24]$ in which behavior responses could induce dramatic changes of dynamical behaviors.

For model (2.5), we have used numerical simulations to find that epidemic peaks can be much reduced or removed, which is very helpful to control influenza outbreaks. For model (2.6), numerical calculations indicate that a slighter mobility response can save a good fraction of susceptible individuals. For example, Figure 4 indicates that 30\% susceptible individuals can be protected from infection when $\alpha=0.1$ at days 120.

Acknowledgements. I am very grateful to two anonymous referees for careful reading and valuable comments which led to improvements of the original manuscript.

Supported by the National Natural Science Foundation of China (11171276) and by the Ministry of Education of China (20100182110003).

\section{References}

[1] M. Balinska, C. Rizzo. Behavioural responses to influenza pandemics: what do we know? PLoS. Curr., (2009), p. RRN1037.

[2] V. Capasso, G. Serio. Ageneralization of the Kermack-McKendrick deterministic epidemic model. Math. Biosci., 42 (1978), 43-61.

[3] J. Cui, Y. Sun, H. Zhu. The impact of media on the control of infectious diseases. J. Dynam. Differential Equations, 20 (2008), 31-53.

[4] W. R. Derrick, P. van den Driessche. A disease transmision model in a nonconstant population. J. Math. Biol., 31 (1993), 495-512

[5] A. d'Onofrio, P. Manfredi. Information-related changes in contact patterns may trigger oscillations in the endemic prevalence of infectious diseases. J. Theor. Biol., 256 (2009), 473-478.

[6] J. M. Epstein, J. Parker, D. Cummings, R. A. Hammond. Coupled contagion dynamics of fear and disease: mathematical and computational explorations. PLoS One, 3 (2008), e3955.

[7] S. Funk, E. Gilad, V. A. A. Jansen. Endemic disease, awareness, and local behavioural response. J. Theor. Biol., 264 (2010), 501-509.

[8] D. Gao, S. Ruan. An SIS patch model with variable transmission coefficients. Mathematical Biosciences, 232 (2011), 110-115.

[9] I. Z. Kiss, J. Cassell, M. Recker, P. L. Simon. The impact of information transmission on epidemic outbreaks. Math. Biosci., 225 (2010), 1-10.

[10] J.P. LaSalle, S. Lefschetz. Stability by Lyapunov's Direct Method. Academic Press, New York, 1961.

[11] W. M. Liu, H. W. Hethcote, S. A. Levin. Dynamical behavior of epidemiological models with nonlinear incidence rates. J. Math. Biol., 25 (1987), 359-380.

[12] W. M. Liu, S. A. Levin, Y. Iwasa. Influence of nonlinear incidence rates upon the behavior of SIRS epidemiological models. J. Math. Biol., 23 (1986), 187-204.

[13] P. Poletti, B. Caprile, M. Ajelli A. Pugliese, S. Merler. Spontaneous behavioural changes in response to epidemics. J. Theor. Biol., 260 (2009), 31-40.

[14] Z. Qiu, Z. Feng. Transmission dynamics of an influenza model with vaccination and antiviral treatment. Bull. Math. Biol., 72 (2010), 1-33.

[15] S. Ruan, W. Wang. Dynamical behavior of an epidemic model with a nonlinear incidence rate. J. Differential Equations, 188 (2003), 135-163.

[16] S. Ruan, W. Wang, S. Levin. The effect of global travel on the spread of SARS. Mathematical Biosciences and Engineering, 3 (2006), 205-218.

[17] L. Sattenspiel, D. A. Herring. Simulating the effect of quarantine on spread of the 1918-19 flue in central Canada. Bull. Math. Biol., 65 (2003), 1-26.

[18] C. Sun, W. Yang, J. Arino, K. Khan. Effect of media-induced social distancing on disease transmission in a two patch setting. Math. Biosci., 230 (2011), 87-95.

[19] M. M. Tanaka, J. Kumm, M. W. Feldman. Coevolution of pathogens and cultural practices: a new look at behavioral heterogeneity in epidemics. Theor. Popul. Biol., 62 (2002), 111-119.

[20] S. Tang, Y. Xiao, Y. Yang, Y. Zhou, J. Wu, Z. Ma. Community-based measures for mitigating the 2009 H1N1 pandemic in China. PLoS One. 5 (2010), e10911.

[21] P. van den Driessche, J. Watmough. Reproduction numbers and sub-threshold endemic equilibria for compartmental models of disease transmission. Math. Biosci., 180 (2002), 29-48.

[22] W. Wang. Epidemic models with nonlinear infection forces. Mathematical Biosciences and Engineering, 3 (2006), $267-279$. 
[23] W. Wang, S. Ruan. Simulating the SARS outbreak in Beijing with limited data. J. Theor. Biol., 227 (2004), 369-379.

[24] D. Xiao, S. Ruan. Global analysis of an epidemic model with nonmonotone incidence rate. Math. Biosci., 208 (2007), 419-429. 